\title{
MABEL, A New Robotic Bipedal Walker and Runner
}

\author{
J.W. Grizzle ${ }^{1}$, Jonathan Hurst ${ }^{2}$, Benjamin Morris ${ }^{3}$, Hae-Won Park ${ }^{4}$, and Koushil Sreenath ${ }^{1}$
}

\begin{abstract}
This paper introduces MABEL, a new platform for the study of bipedal locomotion in robots. One of the purposes of building the mechanism is to explore a novel powertrain design that incorporates compliance, with the objective of improving the power efficiency of the robot, both in steady state operation and in responding to disturbances. A second purpose is to inspire the development of new feedback control algorithms for running on level surfaces and walking on rough terrain. A third motivation for building the robot is science and technology outreach; indeed, it is already included in tours when K-through-12 students visit the College of Engineering at the University of Michigan. MABEL is currently walking at $1.1 \mathrm{~m} / \mathrm{s}$ on a level surface, and a related monopod at Carnegie Mellon is hopping well, establishing that the testbed has the potential to realize its many objectives.
\end{abstract}

\section{INTRODUCTION}

This paper describes MABEL, a new bipedal testbed at the University of Michigan's EECS Department. The robot is designed to be a good walker and an even better runner. It seeks to take advantage of compliance in its powertrain to improve the efficiency and agility of legged locomotion.

MABEL grew out of a collaborative effort between the University of Michigan and the Robotics Institute of Carnegie Mellon University; the acknowledgements attempt to summarize the important contributions made by many people to the conception, design and construction of this robotic testbed. As with many collaborative efforts, each group of participants brought different expectations, motivations and skills to the project. The Carnegie Mellon team was primarily interested in the design of the mechanism, with a focus on dynamic behavior; the Michigan team was looking for an exciting platform to inspire new control designs; the primary sponsor, NSF, was as much interested in the potential of the robot for outreach as it was in the new science that would come of the effort.

Section II overviews the general features of the robot; a much more detailed treatment is available in [18]. Section III summarizes the key features of the nonlinear, hybrid dynamic model of the robot for a walking gait. Initial experimental walking results are described in Sect. IV. Conclusions and future directions are provided in Sect. V. The latest information on the robot can be found at [11].

\footnotetext{
1 Control Systems Laboratory, Electrical Engineering and Computer Science Department, University of Michigan, Ann Arbor, MI 48109-2122, USA. \{grizzle, koushils\}@umich.edu

2 Mechanical Engineering, 204 Rogers Hall, Oregon State University, Corvallis, OR 97331, USA. jonathan.hursteoregonstate.edu

${ }^{3}$ Eaton Innovation Center, 26201 Northwestern Highway, Southfield, MI 48076, USA. benjaminmorris@eaton. com

4 Mechanical Engineering Department, University of Michigan, Ann Arbor, MI 48109-2125, USA. parkhw@umich.edu
}

\section{Testbed OVERVIEW}

\section{A. Bipedal Mechanism}

MABEL is a planar bipedal robot comprised of five links assembled to form a torso and two legs with knees; see Fig. 1. The legs are terminated in point feet. The planar nature of the robot is manifested in the hips, which are constrained to revolute motion in the sagittal plane. The robot is attached to a boom and hence walks or runs in a circle; when the radius is sufficiently large, this approximates well locomotion along a straight line. Many of the components used in the robot are given in Table I.

Up to this point, the robot is similar to RABBIT, the French robot on which the Michigan team collaborated from 2001 through 2004 [4], [29], [30]. The novel aspects of MABEL, and there are many, appear in the transmission or powertrain [19], [18]. First of all, all of the actuators (four DC-brushless motors, two for each leg) are located in the torso, so that the legs are as light as possible; this is done

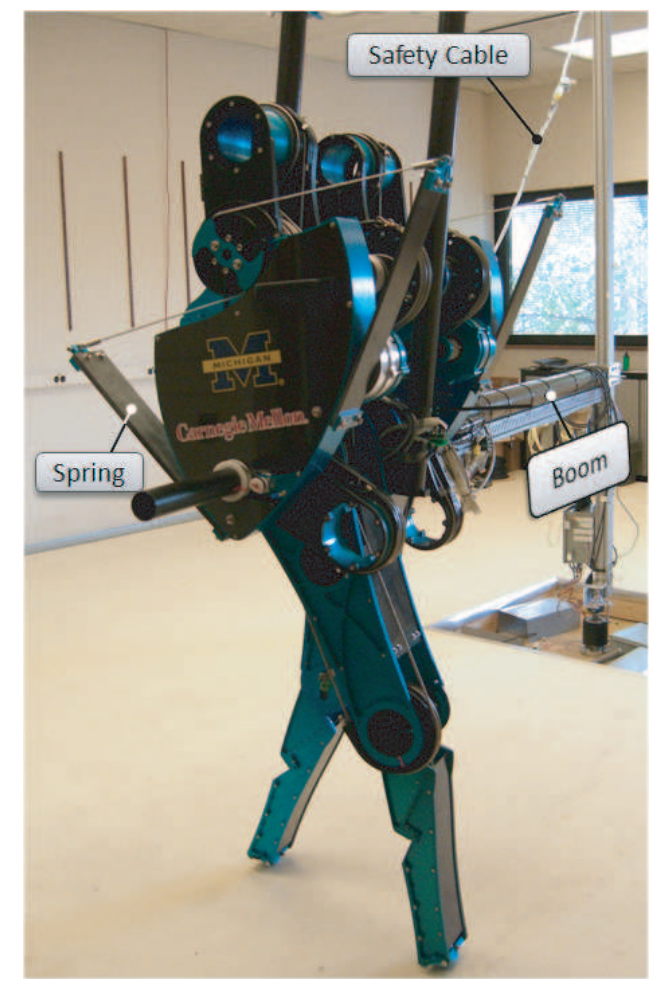

Fig. 1. MABEL, showing the boom that provides stabilization in the frontal plane and a safety cable. The shin and thigh are each $50 \mathrm{~cm}$ long, making the robot one meter tall at the hip. The overall mass is $56 \mathrm{Kg}$. The robot's powertrain incorporates unilateral springs for shock absorption and energy storage; see text and Figs. 2 and 3. 

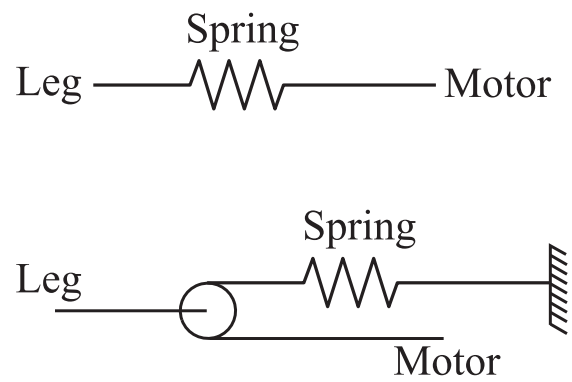

Fig. 2. The top figure shows a series elastic element without a differential: the entire spring moves back and forth. The bottom figure illustrates that with a differential, the spring can remain grounded at one end. MABEL's powertrain is based on the second configuration.

for running efficiency. In RABBIT and most other robots, the knee actuators are mounted on the thigh. Secondly, the actuated degrees of freedom of each leg do not correspond to the knee and the hip angles (the hip angle being the relative angle between the torso and thigh). Instead, for each leg, a collection of differentials is used to connect two motors to the hip and knee joints in such a way that one motor controls the angle of the virtual leg consisting of the line connecting the hip to the toe, and the second motor is connected-in series with a spring-in order to control the length or shape of the virtual leg; see Figs. 2, 3 and 4.

Roughly speaking, the rationale for this design is that it makes the robot a hybrid of RABBIT, a robot that walks extremely well, but never achieved a stable running gait [20], and a Raibert Hopper [25], a robot that "runs" remarkably well ${ }^{1}$. The springs in MABEL serve to isolate the reflected rotor inertia of the leg-shape motors from the impact forces at leg touchdown and to store energy in the compression phase of a running gait, when the support leg must decelerate the downward motion of the robot's center of mass; the energy stored in the spring can then be used to redirect the center of mass upwards for the subsequent flight phase, when both legs will be off the ground [1], [2], [3], [5], [10]. Both of these properties (shock isolation and energy storage) enhance the energy efficiency of running and reduce overall actuator power requirements. This is also true for walking on flat ground, but to a lesser extent, due to the lower forces at leg impact and the reduced vertical travel of the center of mass. The robotics literature strongly suggests that shock isolation and compliance will be very useful for walking on uneven terrain [14], [27], [7], [6], [13], [21], [28].

The third novelty in MABEL's powertrain is that the springs in series with the leg shape motors are unilateral in the sense that they compress, but do not extend beyond their nominal rest length; instead, once a spring reaches its rest length, the position of the leg-shape motor and the shape of the virtual leg are rigidly connected (i.e., directly, through a gear ratio, and no longer through a compliant element); see Fig. 3 for how this is achieved. This is a big advantage in walking where at leg exchange, when the former stance leg

\footnotetext{
${ }^{1}$ A bipedal version of the hopper work is reported in [14].
}

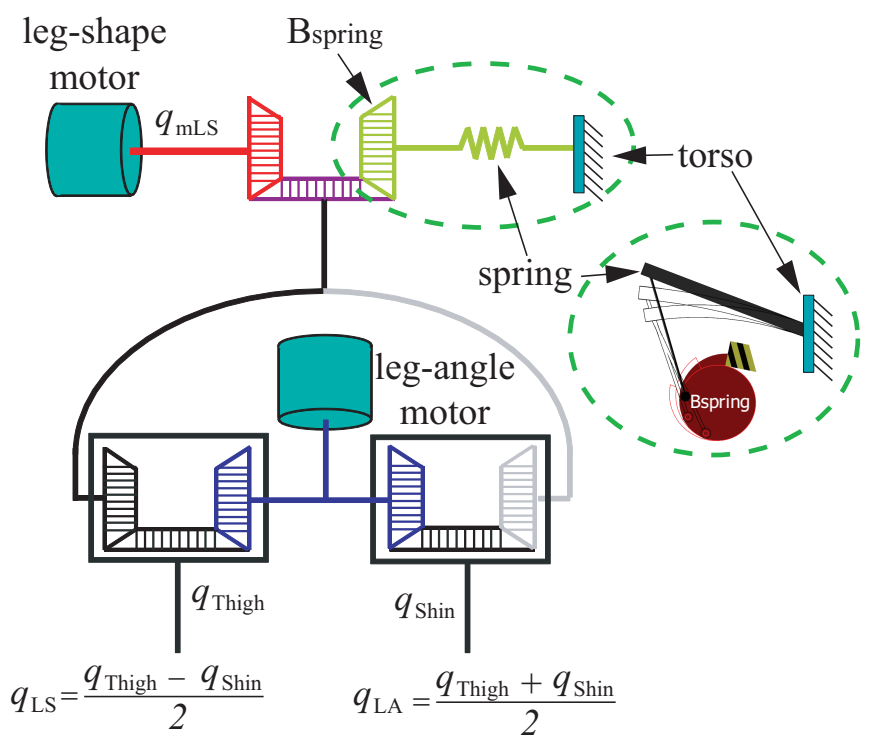

Fig. 3. MABEL's powertrain (same for each leg), all housed in the torso. Two motors and a spring are connected to the traditional hip and knee joints via three differentials. On the robot, the differentials are realized via cables and pulleys [18] and not via gears. They are connected such that the actuated variables are leg angle and leg shape, see Fig. 4, and so that the spring is in series with the leg shape motor, see Fig. 2. The effect of a torsional spring is realized as shown in the expanded view. The base of the spring is grounded to the torso, and the other end is connected to the differential via a cable. Because the spring is connected via cables, it is unilateral. When the spring reaches its rest length, the pulley hits a hard stop, formed by a very stiff damper. When this happens, the leg shape motor is, for all intents and purposes, rigidly connected to leg shape through a gear ratio.

must be lifted from the ground, this motion does not have to "fight" a spring that is trying to extend due to the non-zero mass of the shin. Similarly, a unilateral spring also makes it easier to initiate take-off in running (i.e., transition from stance phase to flight phase). Roughly speaking, the springs are present when they are useful for shock attenuation and energy storage, and absent when they would be a hinderance for lifting the legs from the ground. The springs can be easily changed from one experiment to another in order to study the relation of spring stiffness to gait efficiency or robustness to perturbations, for example. An optional pre-load is easily established on the springs as well, so that compliance only comes into play when sufficiently large forces are present; this is convenient for testing simplified walking strategies while debugging the electronics and software.

A fourth novel feature of the robot is that it is constructed from two monopods joined at the hip. By removing six bolts, half of MABEL's torso and one leg can be removed, yielding a monopod. In fact, Thumper at CMU is literally the left half of MABEL [17].

Remark: The springs in MABEL may seem similar to the MIT series elastic actuator (SEA) [15], [24], [22], [23]. Any resemblance is superficial. The MIT actuator is designed for force control and cannot store significant amounts of energy. MABEL's springs provide a revolute instantiation of a spring-loaded prismatic (pogo-stick) leg. They can easily absorb $150 \mathrm{~J}$ of energy (the equivalent of dropping the robot 


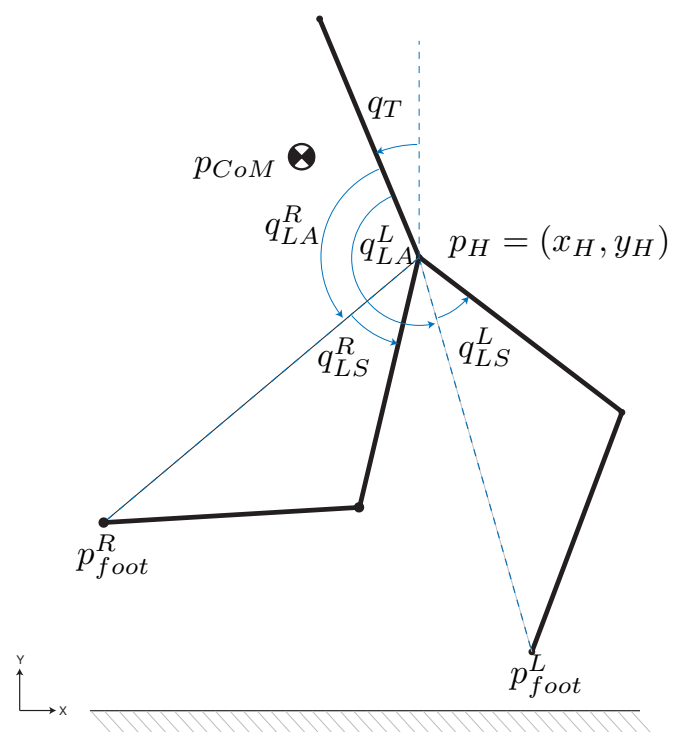

Fig. 4. Coordinate definitions used on the robot are indicated on the figure. The virtual legs are given by the dashed lines connecting the leg ends and the hip. The actuated variables $q_{L A}^{R}, q_{L_{A}}^{L}$ (leg angles) and $q_{L S}^{R}, q_{L S}^{L}$ (leg shapes) correspond to the virtual legs. The length of a virtual is given by $\cos \left(q_{L S}\right)$. The springs in the transmissions (c.f. Fig. 3) make the virtual legs "compliant" in the radial direction, that is, along the dashed lines.

from a height of $30 \mathrm{~cm}$ ). The compliance is designed to play a significant role in the natural dynamics of the system. When MABEL runs, most of the energy of the running gait will be stored and released from the mechanical springs. The spring in the MIT series elastic actuator is several orders of magnitude smaller in size, and is used primarily for filtering and sensing of external forces, rather than energy storage.

There are of course potential drawbacks to having large springs, as on MABEL. First, because of the hard stop near zero force, we cannot control the force as accurately, in both the positive and negative directions, as one can in a series elastic actuator. In addition, because our spring is very large, the behavior of the robot is both enabled and limited by its behavior: as long as we are utilizing the spring by bouncing at approximately its natural frequency, it is a great advantage. However, if we try to create behaviors, such as step changes in force, that are not part of the natural dynamics of a springmass system, performance will be limited. Such limitations are true for all systems with natural dynamics, including motor inertias, and it will be an important issue to keep in mind when designing controllers for the robot.

\section{B. Embedded Control System}

MABEL uses a QNX real-time computing and DAQ environment to acquire data from sensors, compute control action, and output commands to actuators, all at a rate of $1 \mathrm{KHz}$. The software framework for the control system implementation is based on RHexLib, a system architecture originally developed for the RHex running robot [26]. RHexLib is a collection of software libraries that facilitate implementation of real-time controllers, switching of controller modules, over the network data logging and communication with the
TABLE I

COMPONENTS USED IN MABEL

\begin{tabular}{|c|c|c|}
\hline Component & Model & Manufacturer \\
\hline qLA BLDC motors & QBO5600-X0X & \multirow{2}{*}{$\begin{array}{l}\text { Emoteq } \\
\text { Corporation, } \\
\text { Tulsa, OK, USA }\end{array}$} \\
\hline $\mathrm{q}_{\mathrm{LS}}$ BLDC motors & QBO5601-X0X & \\
\hline Motor current drives & PIC-15/200 & $\begin{array}{l}\text { Elmo Motion Con- } \\
\text { trol Ltd., } \\
\text { Petach-Tikva } \\
\text { 49103, Israel }\end{array}$ \\
\hline $\begin{array}{l}\text { Motor and joint } \\
\text { encoders }\end{array}$ & $\begin{array}{l}\text { RMK3 } \\
\text { (2048 counts/rev) }\end{array}$ & \multirow{2}{*}{$\begin{array}{l}\text { RLS d.o.o. } \\
\text { Ljubljana-Dobrunje, } \\
\text { Slovenia }\end{array}$} \\
\hline $\begin{array}{l}\text { Boom and torso } \\
\text { encoders }\end{array}$ & $\begin{array}{l}\text { RM36 } 5 \text { V } \\
\text { (2048 counts/rev) }\end{array}$ & \\
\hline $\begin{array}{l}\text { Digital I/O and } \\
\text { counter timer board }\end{array}$ & PMC464 & \multirow[t]{3}{*}{$\begin{array}{l}\text { Acromag Incorporate } \\
\text { Wixom, MI, USA }\end{array}$} \\
\hline Counter timer board & PMC482 & \\
\hline PCI analog $\mathrm{I} / \mathrm{O}$ card & PMC730 & \\
\hline Real-time controller & $\begin{array}{l}\text { CP-306-V-TR2 } \\
(2 \mathrm{GHz} \text { Celeron M) }\end{array}$ & $\begin{array}{l}\text { Kontron, } \\
\text { Kaufbeuren, } \\
\text { Germany }\end{array}$ \\
\hline
\end{tabular}

robot, and in providing a user-interface for monitoring the robot state on a secondary Linux-based system.

\section{Mathematical Model}

Walking is modeled as a hybrid system corresponding to the alternation of phases of single support (one leg in contact with the ground) and double support (both legs in contact with the ground), where the double support phase is instantaneous, and no slipping occurs during the single support phase. The most general form of the robot model is discussed first. Important special cases, such as those arising when ground contact conditions create holonomic constraints at the leg ends, are presented next, followed by the impact model. The components are then assembled into an overall hybrid model.

\section{A. 9 DOF model}

A planar mechanism consisting of five rigid links connected via revolute joints in a tree structure with no contacts or constraints has seven DOF: a degree of freedom associated with the orientation of each link, plus two DOF associated with the horizontal and vertical displacement of the center of mass within the sagittal plane. The springs present in MABEL's powertrain provide two additional degrees of freedom, taken here as the leg-shape motor positions, measured relative to the torso, for the left and right legs, $q_{m L S}^{L}$ and $q_{m L S}^{R}$, for a total of nine DOF. The state vector of the dynamical model is thus 18-dimensional: there are nine configuration variables required to describe the position of the robot, plus the associated velocities.

A convenient choice of link configuration variables is depicted in Fig. 4, namely, the left and right leg angles (the relative angles between the torso and the virtual legs connecting the hip to the toes), $q_{L A}^{L}$ and $q_{L A}^{R}$, the left and right leg shape angles (the relative angles of the virtual leg 
with respect to the thigh), $q_{L S}^{L}$ and $q_{L S}^{R}$, the angle of the torso with respect to the vertical, $q_{T}$, and the cartesian position of the hips, $\left(x_{H}, y_{H}\right)$. The overall generalized configuration $q_{e}$ is formed by appending to this $q_{m L S}^{L}$ and $q_{m L S}^{R}$.

The dynamic model is easily obtained with the method of Lagrange $^{2}$ if the springs in the powertrain are replaced with generalized forces, which can then be specified in a second step. Denoting the Lagrangian as $L=K-V$, a second order dynamical model immediately follows

$$
\frac{d}{d t} \frac{\partial L}{\partial \dot{q}_{e}}-\frac{\partial L}{\partial q_{e}}=\Gamma_{e},
$$

where $\Gamma_{e}$ is the vector of generalized forces and torques applied to the robot. It is standard to write the model in the form

$$
D_{e}\left(q_{e}\right) \ddot{q}_{e}+C_{e}\left(q_{e}, \dot{q}_{e}\right) \dot{q}_{e}+G_{e}\left(q_{e}\right)=\Gamma_{e}
$$

where, $D_{e}$ is the inertia matrix, the matrix $C_{e}$ contains Coriolis and centrifugal terms, and $G_{e}$ is the gravity vector.

The vector of generalized forces and torques can be expressed as

$$
\Gamma_{e}=B_{e} u+E_{e}\left(q_{e}\right) F^{e x t}+\tau_{f r i c}\left(q_{e}, \dot{q}_{e}\right)+\tau_{s p r}\left(q_{e}, \dot{q}_{e}\right),
$$

where the matrices $B_{e}$ and $E_{e}$ are derived from the principle of virtual work and define how the joint torques, $u$, and external forces at the leg end, $F^{e x t}$, enter the model, and $\tau_{\text {fric }}$ represents joint friction. These terms are standard [9] and are not discussed further for reasons of space.

The term $\tau_{s p r}\left(q_{e}, \dot{q}_{e}\right)$ is the vector of torques acting on the left and right spring differentials. The following discussion applies to each side of the robot. Let $B_{\text {spring }}$ denote the angular position of the differential to which the spring is attached, which can be expressed in terms of $q_{m L S}$ and $q_{L S}$; see Fig. 2. Assume $B_{\text {spring }}=0$ corresponds to the rest position of the spring. Then,

$$
\tau_{\text {spr }}\left(q_{e}, \dot{q}_{e}\right)=\left\{\begin{array}{lr}
K_{\text {sp }} B_{\text {spring }}, & \text { if } B_{\text {spring }} \geq 0 \\
K_{\text {damp }} B_{\text {spring }}+D_{\text {damp }} \dot{B}_{\text {spring }} \\
\text { otherwise, }
\end{array}\right.
$$

where $K_{s p}$ is the effective radial spring stiffness, and the coefficients of the damper forming the "hard stop" are $K_{\text {damp }}$ and $D_{\text {damp }}$.

Remark: We sometimes also model the $B_{\text {spring }}$ pulley hitting the hard stop as a rigid impact.

\section{B. Lower DOF models}

When the robot is in single support, that is, one leg is on the ground and it is neither slipping nor rebounding, the foot acts like a pin joint. In this case, the hip coordinates are no longer independent of the other coordinates and can be eliminated, resulting in a seven DOF model. This is standard and is not discussed further.

\footnotetext{
${ }^{2}$ The model of the differentials in the powertrain is somewhat involved. A symbolic file that computes the Lagrangian will be made available online at [11]. The inertias of the motor rotors as well as the pulleys in the differentials and the effective gear ratios achieved by passing cables over pulleys of varying radii are all accounted for in this model.
}

The springs in the robot have to be stiff enough to support the weight of the robot, reflected through the leg joints and transmission. The mass of the shins is small in relation to the overall mass of the robot. Consequently, the spring of the swing leg deflects very little during the stance phase, and it is computationally advantageous to eliminate this degree of freedom when designing walking motions for the robot, resulting in a six degree of freedom model when in single support. Denote the resulting configuration variables by $q$. Letting $x=(q, \dot{q})$ and $u$ the motor torques, the stance-phase model is then easily expressed in state variable form

$$
\dot{x}=f(x)+g(x) u \text {. }
$$

\section{Impact model and leg swapping}

Due to the rigid links used in MABEL, a rigid impact model is appropriate when the swing leg end touches the walking surface [8], [16]. It is assumed that the contact of the swing leg end with the ground results in no rebound and no slipping of the swing leg, and the stance leg lifting from the ground without interaction (see [31, pp. 49] for a complete set of hypotheses). This results in the double support phase being instantaneous. The ground reaction forces are represented by impulses, leading to an instantaneous change in the velocity coordinates, but there is no instantaneous change in the positions.

Following the development in [31, pp. 55], the nine degree of freedom dynamic model (1) is used to determine an expression for $\dot{q}^{+}$, the vector of angular velocities just after impact, in terms of the configuration of the robot at impact and $\dot{q}^{-}$, the vector of angular velocities just before impact [8], [16]. The post-impact velocity is then used to re-initialize the stance-phase model for the next step. The stance-phase model must make a choice of the stance leg (i.e., left or right). In order to re-use the same model, a change of coordinates is used so that the former swing leg becomes the stance leg and vice versa [31, pp. 57]. It is convenient to include this coordinate swap as part of the impact map. The final result is an expression for $x^{+}=\left(q^{+}, \dot{q}^{+}\right)$in terms of $x^{-}=\left(q^{-}, \dot{q}^{-}\right)$, which is written as

$$
x^{+}=\Delta\left(x^{-}\right) \text {. }
$$

Remark: Just before impact, the spring of the stance leg is still supporting the weight of the robot and hence the $B_{\text {spring }}$ pulley position is non-zero. As part of the impact and leg swapping map, $B_{\text {spring }}$ is set equal to zero. This represents energy loss (typically small on a well-designed gait) and, in any case, approximates the negative work that the leg shape motor would have to do in order to begin leg retraction.

Remark: Due to the springs in the powertrain, the reflected inertias of the leg shape motors are effectively isolated from the impact. They are only coupled to the impact through the relatively small inertia of the spring differentials; see Fig. 3. 


\section{Hybrid model of walking}

The dynamic model (4) and the impact model (5) together yield a nonlinear system with impulse effects [31],[32]

$$
\left\{\begin{aligned}
\dot{x} & =f(x)+g(x) u, & & x^{-} \notin \mathcal{S}, \\
x^{+} & =\Delta\left(x^{-}\right), & & x^{-} \in \mathcal{S},
\end{aligned}\right.
$$

where $\mathcal{S}$ is the surface of points where the swing leg height above the ground is zero and the swing leg is strictly in front of the stance leg.

\section{Initial Walking Gait Design}

This section presents an elementary walking gait. Its purpose is simply to demonstrate that the testbed is functional. The control law is not sophisticated enough to properly take advantage of the compliance in the powertrain. That is a subject of ongoing work, which will be presented elsewhere.

A walking gait was designed using the method of virtual constraints. Virtual constraints are holonomic constraints among the generalized coordinates of the robot that are dynamically imposed through feedback control ${ }^{3}$ [12]. Their function is to coordinate the evolution of the various links throughout a step, with the goal of achieving a closed-loop mechanism that naturally gives rise to a periodic walking gait. The most direct form of the constraint is

$$
y=h(q)=q_{a}-h_{d}(\theta),
$$

${ }^{3} \mathrm{~A}$ feedback controller driving $y$ to zero, $y=h(q)=0$, results in $q_{a}=$ $h_{d}(\theta)$, which is a holonomic constraint on the evolution of the dynamics. An important difference with the classical notion of a (physical) holonomic constraint is that the feedback torques used to impose the virtual constraint (i.e., zero y) do mechanical work on the system; see [31].
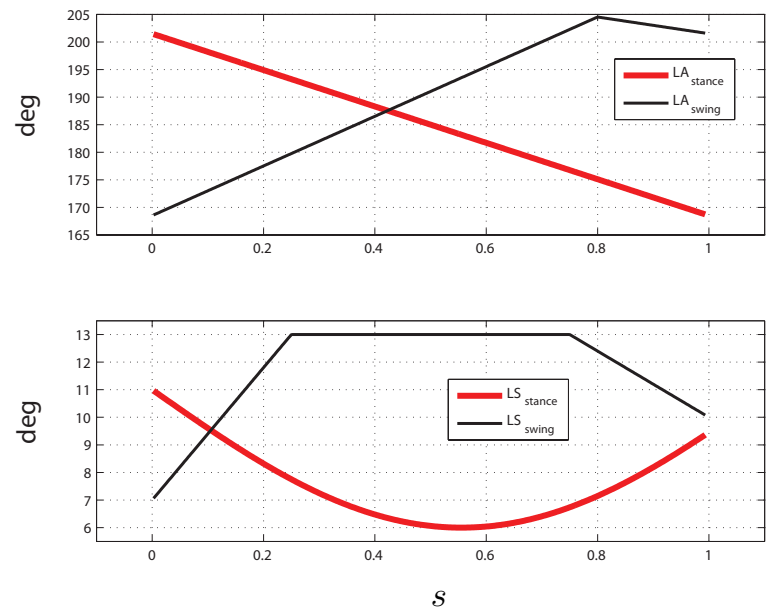

Fig. 5. The hand-crafted virtual constraints used in the walking experiment, expressed as a function of $s$, the normalized quantity used to parameterize the robot's position within each step. There are four constraints because the robot has four actuators. The stance leg angle constraint keeps the torso upright as the robot progresses in the step, while the stance leg shape constraint straightens the leg toward mid step and shortens it to prepare for impact at the end of the step. The swing leg shape constraint folds the knee for clearance and straightens the leg to prepare for impact. The swing leg angle constraint overshoots the desired final position to avoid toe stubbing.
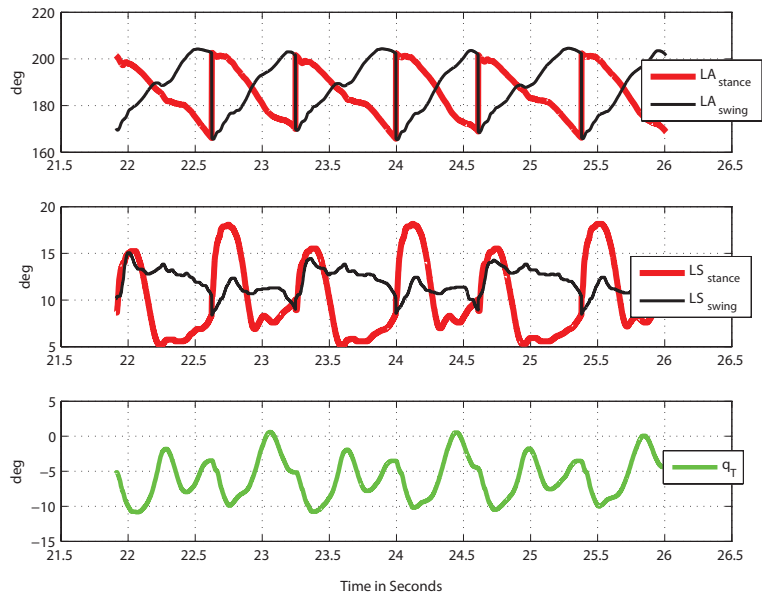

Fig. 6. Joint positions (degrees) versus time (seconds) for steps 30 to 36 of the experiment. The vertical lines in the LA plots mark the impact points, that is, the transition from one stance leg to the other. There is a notable amount of asymmetry in the gait with respect to left and right legs of the robot, with this most clearly showing up in the trace of the torso angle. The large deviations in torso angle are occurring when the right leg is stance.
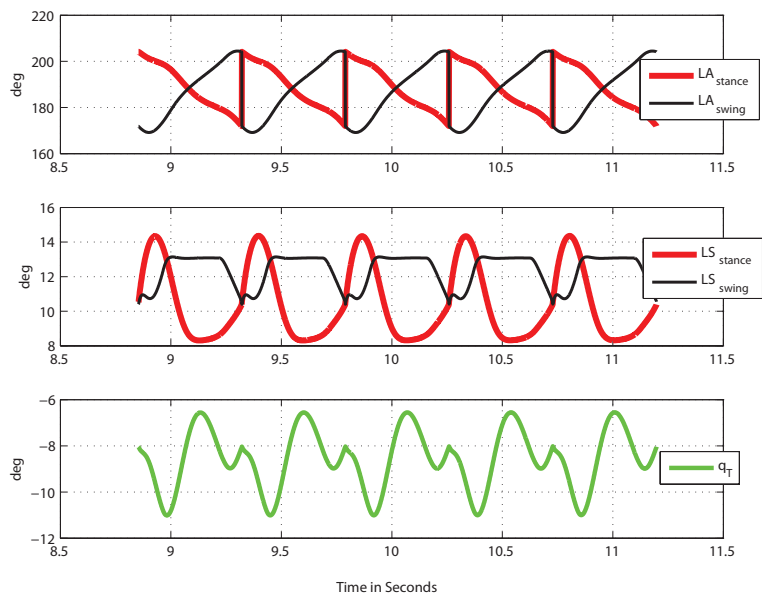

Fig. 7. Joint positions (degrees) versus time (seconds) for steps 20 to 25 of the simulation model. The vertical lines in the LA plots mark the impact points, that is, the transition from one stance leg to the other. The traces correspond reasonably well to the experimental values, with the exception of the torso trace when the right leg is in stance.

where $q_{a}=\left[q_{L A}^{L}, q_{L A}^{R}, q_{L S}^{L}, q_{L S}^{R}\right]^{\prime}$ is the vector of actuated coordinates, $\theta=\theta(q)$ is a quantity that is strictly monotonic (i.e., strictly increasing or decreasing) along a typical walking gait, and $h_{d}(\theta)$ is the desired evolution of the actuated variables as a function of $\theta$. Roughly speaking, $\theta$ is used to replace time in parameterizing a periodic motion of the biped. For MABEL, an appropriate choice is (a linear approximation of) the angle of the line connecting the stance toe to the robot's center of mass, which is taken here as

$$
\theta=q_{T}+q_{L A}^{\text {Stance }},
$$

where $q_{L A}^{\text {Stance }}$ is equal to $q_{L A}^{L}$ or $q_{L A}^{R}$, depending on which is the current stance leg. The angle $\theta$ is normalized to the interval $[0,1]$ and called $s$. 
Because parameter identification of the dynamic model had not been completed at the time of these experiments, the virtual constraints were designed by hand instead of being designed on the basis of the dynamic model (6), as in [31] and [29]. Simulation results from the current version of the model are shown below nevertheless, for comparison purposes. Virtual constraints were designed for leg angle and leg shape, for both the stance phase and swing phase of the gait. The constraints used are depicted in Fig. 5. The purpose the stance leg angle constraint is to regulate the horizontal position of the torso center of mass throughout the step; this has a large affect on walking speed. The constraint on swing leg angle brings the leg forward, preparing it for impact with a desired step length. The swing leg shape is responsible for lifting the swing leg from the ground and avoiding foot scuffing. The stance leg shape was selected so as to achieve an approximate parabolic trajectory for the vertical height of the hip. The exact values chosen were arrived at on the basis of experience with a related robot, RABBIT [4], and a modest amount of trial and error.

The virtual constraints were applied to the robot in an approximate manner, as in [30], via PD controllers. The controllers on leg angle acted on the error of the measured leg angle and the desired angle given by the virtual constraint, while the controllers for leg shape, due to the presence of the springs, used an inner-outer loop configuration, with the inner loop applied to leg shape motor position and the outer loop to leg shape. The result was a stable gait in which MABEL took 45 steps before being stopped by the experimenters.

The experiment began with MABEL elevated in the air. The legs were servoed to a nominal double support position corresponding to $s=0$. The robot was then placed on the ground. The closed-loop system is asymptotically stable in double support with zero forward velocity $(\dot{s} \equiv 0)$. To initiate walking, an external force was applied to MABEL by means of pushing on the boom which provides its support in the frontal plane. Application of this force provided the robot with an initial forward velocity sufficient to enter the basin of attraction of the controller. The result was a stable walking gait with average forward speed of $0.89 \mathrm{~m} / \mathrm{s}$ (other gaits have been implemented on the robot, ranging in speed from 0.48 $\mathrm{m} / \mathrm{s}$ to $1.1 \mathrm{~m} / \mathrm{s}$ ). A stick figure representation of the gait is shown in Fig. 8. For videos of MABEL walking, see [11].

Figure 6 depicts a snapshot the evolution of the robot's links through out the gait. For comparison purposes, the evolution predicted by the simulation model is shown in Fig. 7. The comparison is made further in Fig. 9. An important difference between the simulation and experiments is the left-right asymmetry that is currently present in the physical mechanism. If this cannot be eliminated on the testbed, then it will have to be included in the model.

\section{Conclusions and Future Challenges}

MABEL, a planar, bipedal robot testbed has been installed at the University of Michigan. The robot has a novel powertrain, based on cable differentials and springs, so that the virtual legs represented by the lines connecting the hip to the toes are compliant in the radial direction. This design seeks to improve power efficiency and augment the ability of the robot to absorb shocks at leg impact.

MABEL is currently walking at $1.1 \mathrm{~m} / \mathrm{s}$ on a level surface. The utility of the testbed will be proven when the robot is able to run well on flat ground and walk well on uneven ground. We are working hard to develop the control laws that will allow the robot to meet these ambitious objectives.

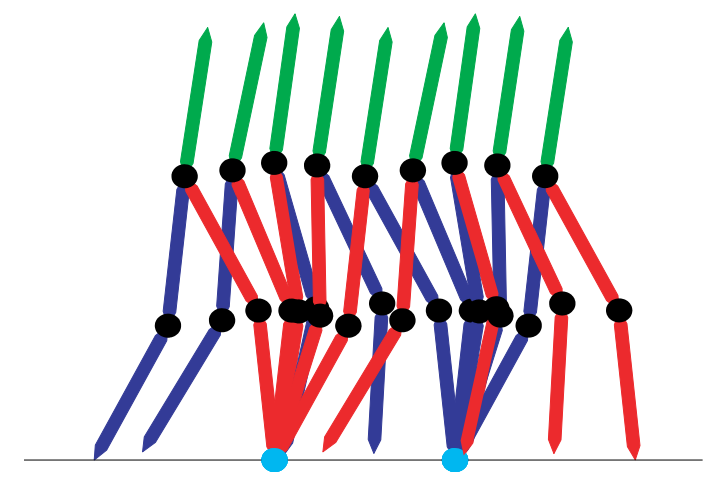

Fig. 8. Stick figure of the robot walking at $0.89 \mathrm{~m} / \mathrm{s}$, on the basis of the virtual constraints of Fig. 5.

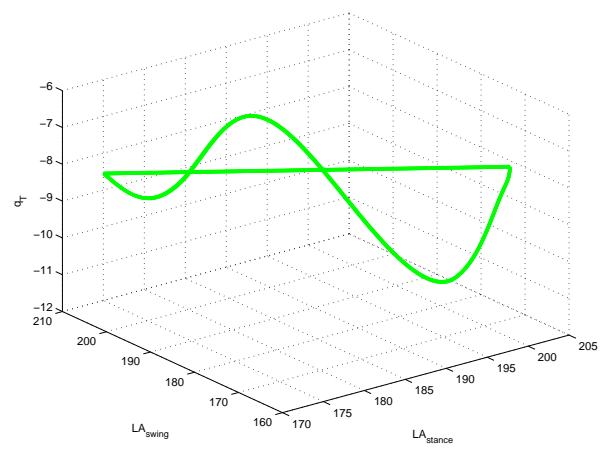

(a) simulation

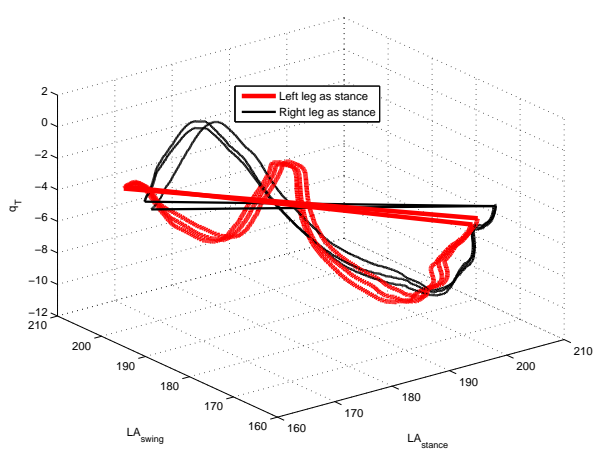

(b) experiment

Fig. 9. A 3-D projection of the limit cycle predicted by the model in (a) versus the limit cycle achieved in the experiment (b). The lower plot highlights the left-right asymmetry present in the current set up of the testbed. The source of the asymmetry is unknown at the moment. 
ACKnowledgment: In Fall of 2004, J.W. Grizzle and A. Rizzi (then of the Robotics Institute, CMU, currently with Boston Dynamics) initiated an NSF-supported project to build a bipedal walker and runner incorporating compliance. The general features of the robot (morphology, dimensions, target mass and motor sizing) were set by J.W. Grizzle, J. Hurst, B. Morris, and A. Rizzi, on the basis of preliminary modeling and control law studies. The detailed mechanical design of the biped, MABEL, and of the associated monopod, Thumper, was done solely by J. Hurst, who also oversaw the machining of the robots' parts. J. Chestnutt led the real-time control platform development, with valuable support provided by C. Haynes and P. Velagapudi. B. Morris initiated the transition of software support from CMU to Michigan and expanded the coding scope to address both Acromag IO driver interfaces and the realtime softwarein-the-loop evaluation of potential control algorithms. G. Buche, J. Chestnutt, B. Kirby, J. Hurst, and P. Velagapudi contributed to the design of the electronics, power supply and safety interlock system. The assembly of MABEL at Michigan, the development of the laboratory housing the robot, the commissioning and debugging of the overall system, and the development and implementation of the control laws demonstrated here were conducted by H.W. Park, K. Sreenath, and J.W. Grizzle, with the help of G. Buche, L. Dicken, J. Koncsol, B. Morris, S. Platt, I. Poulakakis, and S. Zhang.

The work of J.W. Grizzle, B. Morris, H.W. Park, and K. Sreenath was supported in part by NSF grant ECS0600869, and in part by the University of Michigan College of Engineering. The work of J. Hurst was supported by an NSF fellowship, an IGERT fellowship, and by Carnegie Mellon University.

\section{REFERENCES}

[1] R. Alexander, "Three uses for springs in legged locomotion," International Journal of Robotics Research, vol. 9, no. 2, pp. 53-61, 1990.

[2] R. Blickhan, "The spring-mass model for running and hopping," vol. 22, no. 11-12, pp. 1217-1227, 1989.

[3] G. A. Cavagna, N. C. Heglund, and R. C. Taylor, "Mechanical work in terrestrial locomotion: Two basic mechanisms for minimizing energy expenditure," American Journal of Physiology, vol. 233, no. 5, pp. R243-R261, 1977.

[4] C. Chevallereau, G. Abba, Y. Aoustin, E. Plestan, F. Westervelt, C. Canduas-de Wit, and J. Grizzle, "RABBIT: A testbed for advanced control theory," IEEE Control Systems Magazine, vol. 23, no. 5, pp. 57-79, October 2003.

[5] C. Chevallereau, E. Westervelt, and J. Grizzle, "Asymptotically stable running for a five-link, four-actuator, planar bipedal robot," International Journal of Robotics Research, vol. 24, no. 6, pp. $431-464$, June 2005.

[6] M. A. Daley and A. A. Biewener, "Running over rough terrain reveals limb control for intrinsic stability," Proceedings of the National Academy of Sciences of the United States of America, vol. 103, no. 42, pp. 15681-15686, October 2006.

[7] M. A. Daley, J. R. Usherwood, G. Felix, and A. A. Biewener, "Running over rough terrain: guinea fowl maintain dynamic stability despite a large unexpected change in substrate height," The Journal of Experimental Biology, vol. 209, pp. 171-187, 2006. [Online]. Available: http://jeb.biologists.org/cgi/content/full/209/1/171/DC1

[8] A. Formal'sky, Locomotion of Anthropomorphic Mechanisms, ser. Nauka. Moscow: in Russian, 1982.
[9] Y. Fujimoto and A. Kawamura, "Simulation of an autonomous biped walking robot including environmental force interaction," IEEE Robotics and Automation Magazine, pp. 33-42, June 1998.

[10] R. Full and D. Koditschek, "Templates and anchors: Neuromechanical hypotheses of legged locomotion on land," Journal of Experimental Biology, vol. 202, pp. 3325-3332, December 1999.

[11] J. Grizzle, "Publications on robotics and control," www.eecs.umich. edu/ grizzle/papers/robotics.html, September 2008.

[12] J. Grizzle, G. Abba, and F. Plestan, "Asymptotically stable walking for biped robots: Analysis via systems with impulse effects," IEEE Transactions on Automatic Control, vol. 46, pp. 51-64, January 2001.

[13] K. Hashimoto, Y. Sugahara, H. Sunazuka, C. Tanaka, A. Ohta, M. Kawase, H. Lim, and A. Takanishi, "Biped landing pattern modification method with nonlinear compliance control," May 2006, pp. 1213-1218.

[14] J. Hodgins and M. H. Raibert, "Adjusting step length for rough terrain locomotion," IEEE Transactions on Robotics and Automation, vol. 7(3), pp. 289-298, June 1991.

[15] J. Hollerbach, I. Hunter, and J. Ballantyne, A comparative analysis of actuator technologies for robotics, ser. MIT Press. Khatib, O. and Canny, J., and Lozano-Perez, T., 1992.

[16] Y. Hurmuzlu and D. Marghitu, "Rigid body collisions of planar kinematic chains with multiple contact points," International Journal of Robotics Research, vol. 13, no. 1, pp. 82-92, 1994.

[17] J. Hurst, "The electric cable differential (ECD) leg, http://www.ri. cmu.edu/projects/project_597.html," August 2008.

[18] J. W. Hurst, "The role and implementation of compliance in legged locomotion," Ph.D. dissertation, Robotics Institute, Carnegie Mellon Univesity, August 2008.

[19] J. W. Hurst, J. E. Chestnutt, and A. A. Rizzi, "Design and philosophy of the BiMASC, a higly dynamic biped," in Proceedings of the IEEE International Conference of Robotics and Automation, Roma, Italy, Apr. 2007, pp. 1863-1868.

[20] B. Morris, E. Westervelt, C. Chevallereau, G. Buche, and J. Grizzle, Fast Motions Symposium on Biomechanics and Robotics, ser. Lecture Notes in Control and Information Sciences. Heidelberg, Germany: Springer-Verlag, 2006, ch. Achieving Bipedal Running with RABBIT: Six Steps toward Infinity, pp. 277-297.

[21] M. Ogino, H. Toyama, and M. Asada, "Stabilizing biped walking on rough terrain based on the compliance control," IEEE/RSJ International Conference on Intelligent Robots and Systems, pp. 4047-4052, 29 2007-Nov. 22007.

[22] G. Pratt, "Legged robots at MIT: what's new since Raibert?" IEEE Robotics and Automation Magazine, September 2000.

[23] J. Pratt, M. Chee, A. Torres, P. Dilworth, and G. Pratt, "Virtual model control: an intuitive approach for bipedal locomotion," International Journal of Robotics Research, vol. 20, no. 2, pp. 129-143, February 2001.

[24] J. Pratt and G. Pratt, "Exploiting natural dynamics in the control of 3d bipedal walking simulation," in CLAWAR99, Portsmouth, U.K., September 1999.

[25] M. H. Raibert, Legged robots that balance. Mass.: MIT Press, 1986.

[26] U. Saranli, M. Buehler, and D. Koditschek, "RHex: A Simple and Highly Mobile Hexapod Robot," The International Journal of Robotics Research, vol. 20, no. 7, p. 616631, 2001.

[27] U. Saranli, M. Buehler, and D. E. Koditschek, "Rhex: A simple and highly mobile hexapod robot," International Journal of Robotics Research, vol. 20, no. 7, pp. 616 - 631, July 2001.

[28] T. TAKUMA, S. HAYASHI, and K. HOSODA, "3D Bipedal Robot with Tunable Leg Compliance Mechanism for Multi-modal Locomotion," in Intelligent Robots and Systems, 2008. IROS 2008. IEEE/RSJ International Conference on, 2008, pp. 1097-1102.

[29] E. Westervelt, J. Grizzle, and D. Koditschek, "Hybrid zero dynamics of planar biped walkers," IEEE Transactions on Automatic Control, vol. 48, no. 1, pp. 42-56, January 2003.

[30] E. Westervelt, G. Buche, and J. Grizzle, "Experimental validation of a framework for the design of controllers that induce stable walking in planar bipeds," International Journal of Robotics Research, June 2004.

[31] E. Westervelt, J. Grizzle, C. Chevallereau, J. Choi, and B. Morris, Feedback Control of Dynamic Bipedal Robot Locomotion, ser. Control and Automation. Boca Raton: CRC Press, June 2007.

[32] H. Ye, A. Michel, and L. Hou, "Stability theory for hybrid dynamical systems," IEEE Transactions on Automatic Control, vol. 43, no. 4, pp. 461-474, April 1998. 